\title{
Testable implications of Transferable Utility
}

\author{
Pierre-Andre Chiappori*
}

January 2007

\begin{abstract}
Although the Transferable Utility (TU) assumption is widely used in micro theory, little is known about its testable consequences, and in particular how one can, from the observation of a group's demand, test whether the TU assumption is satisfied within this group. We derive a set of necessary and sufficient conditions on demand that characterize the TU context. The conditions are of two types. First, TU requires the group to behave as a single decision maker. Secondly, within the unitary setting, the representative utility generated by a TU framework must be of the Generalized Quasi Linear (GQL) form. We derive testable properties of the demand function that fully characterize GQL utilities. These additional restrictions only involve specific prices namely, those of the goods that are publicly consumed within the household.
\end{abstract}

\section{Introduction}

The analysis of group behavior is one of the most important topics in microeconomic theory. While some approaches adopt a general viewpoint, ${ }^{1}$ a majority of studies refer to a specific framework, in which utilities are assumed to be transferable within the group. The transferable utility (TU) assumption has a number of distinctive advantages. First, it displays convenient aggregation properties. Specifically, the group aggregate demand can, under $\mathrm{TU}$, be derived from the maximization of a unique, representative utility - a property that greatly simplifies comparative statics investigations. Secondly, the analysis of welfare issues is drastically simplified by the TU assumption. Under TU, indeed, decisions by individual members may affect the location of the group's Pareto frontier, but not its shape (which is always a straight line of slope -1). Under mild normality assumptions, it follows that the intragroup distribution of property rights on the surplus does not affect the group's

*Economics Department, Columbia University, 1009A International Affairs Building, MC 3308, 420 West 118th Street, New York, NY 10027. E-Mail: pc2167@columbia.edu.

${ }^{1}$ See for instance Chiappori and Ekeland $(2006,2007)$. 
decisions; agents always behave so as to maximize the size of the Pareto set. Strong normative conclusions follow from this fact; one may mention, among many examples, the various versions of the celebrated Coase theorem (Coase 1960), Becker's 'Rotten Kid' proposition, or Becker's influential analysis of divorce, which concludes that divorce laws should not affect divorce probabilities. ${ }^{2}$ Thirdly, the TU assumption is remarkably helpful for the analysis of matching and group formation. For instance, in the simple, 'heterosexual' model of one-to-one matching, TU guarantees that any stable match maximizes the sum of surpluses over the population. Not only do existence and (generic) uniqueness follow, but the computation of the corresponding imputations of the surplus are greatly simplified. ${ }^{3}$

Clearly, all these advantages come at a cost: the TU assumption is indeed restrictive. Surprisingly, however, in regard of the paramount role of transferable utility in micro theory, little is known on the exact nature of the corresponding restrictions. Assume, specifically, that one can observe the group's behavior - which takes the standard form of an (aggregate) demand function satisfying some global budget constraint. Is it the case that any demand function is compatible with a TU framework? If not, how is it possible to test, from demand data, whether the TU assumption is satisfied within the group under consideration?

The goal of this note is to provide a comprehensive answer to these questions. We derive a set of necessary and sufficient conditions on demand that characterize the TU context. Specifically, any demand function that stems from the (efficient) demand of a group in which the TU assumptions hold true must satisfy these properties. Conversely, any demand fulfilling these properties can be derived from a TU framework, so that the group under consideration, whatever its structure and decision process, is observationally equivalent to one in which the TU assumption is satisfied.

The conditions are of two types. First, as is well known, TU requires the group to behave as a single decision maker; therefore, all the properties of a standard, 'unitary' demand - income pooling, Slutsky symmetry and negativeness - must be satisfied. Secondly, within the unitary setting, the representative utility generated by a TU framework exhibits specific features. Specifically, TU has been known since Bergstrom (1989) to generate a household utility of the Generalized Quasi Linear (GQL) form. When all commodities are privately consumed within the group, GQL boils down to standard quasi linearity. This implies that the income elasticity of the demand for all goods but one is nil - a highly counterfactual prediction. The existence of commodities that are publicly consumed within the household significantly relaxes the requirements. In practice, we derive a set of testable properties of the demand function that fully characterize GQL utilities. Interestingly enough, these additional restrictions only involve specific prices - namely, those of the publicly consumed goods. In particular, if data do not exhibit (sufficient) variations in these prices, then any framework that is compatible with the unitary setting is also compatible with a TU

\footnotetext{
${ }^{2}$ See Becker $(1992,1993)$, and Chiappori, Iyigun and Weiss (2006) for a recent reconsideration.

${ }^{3}$ See for instance Roth and Sottomayor (2003).
} 
framework.

\section{Transferable utility: a general framework}

\subsection{Preferences}

We consider a general model of group behavior with an arbitrary number of private and public consumption goods. Specifically, we consider a group of $S$ individuals purchasing on a market and consuming $n$ private and $N$ public commodities. ${ }^{4}$ Let $x_{m}=\left(x_{m}^{1}, \ldots, x_{m}^{n}\right)$, where $m=1, \ldots, S$, denote member $m$ 's private consumption and $p=\left(p^{1}=1, \ldots, p^{n}\right)$ the corresponding price vector where $p^{1}$ is normalized to 1 . Similarly, $X=\left(X^{1}, \ldots, X^{N}\right)$ denotes the group's public consumption purchased at price $P=\left(P^{1}, \ldots, P^{N}\right)$. Finally, $y_{m}$ denotes member $m$ 's income $(m=1, \ldots, S)$, and let $y=\sum_{s} y_{s}$ be the group's aggregate income; the group's budget constraint is thus:

$$
\sum_{i, m} p^{i} x_{m}^{i}+\sum_{j} P^{j} X^{j}=\sum_{s} y_{s}=y
$$

We first remind the definition of transferable utility:

Definition 1 The framework defined above satisfies the transferable utility (TU) property on some open set $\mathcal{O}$ of price-income bundles if the following property is satisfied: for each agent $m$, there exist a cardinal representation $u_{m}$ of $m$ 's utility such that for each $(p, P)$ in $\mathcal{O}$, the Pareto set defined by the budget constraint (1) is the hyperplane defined by

$$
\sum_{m} u_{m}=1
$$

Necessary and sufficient conditions for transferability have been known for some time. Specifically, define the notion of general quasilinearity as follows:

Definition 2 The utility functions $u_{m}$ is generalized quasi linear (GQL) if there exists increasing functions $F_{m}, b_{m}$ and increasing, concave functions $A_{m}$ such that:

$$
u_{m}\left(x_{m}, X\right)=F_{m}\left[A_{m}\left(x_{m}^{2}, \ldots, x_{m}^{n}, X\right)+x_{m}^{1} b_{m}(X)\right], \quad m=1, \ldots, S .
$$

Obviously, whenever we are interested in the properties of individual or group demands generated by such utilities, we may assume that $F_{m}$ is the identity transform. ${ }^{5}$

In a seminal contribution, Bergstrom (1989) has shown that transferable utility (TU) requires three properties:

\footnotetext{
${ }^{4}$ The goods are public within the group only; they are privately purchased on the market. One may think of housing or expenditures on children as typical examples.

${ }^{5}$ The requirement that the $A_{m}$ and $b_{m}$ be both increasing is needed for consistency. Indeed, if $b_{m}$ could be decreasing, then $u_{m}$ would also be decreasing for sufficiently large values of $x_{m}^{1}$. And similarly, if $A_{m}$ could be decreasing in its arguments, then $u_{m}$ would also be decreasing for sufficiently small values of $x_{m}^{1}$.
} 
1. individual utilities are of the generalized quasi linear form

2. the $b_{m}(X)$ functions are identical across agents: $b_{1}(X)=\ldots=b_{S}(X)=b(X)$

3. the allocation of resources between members is such that each member has a positive consumption of commodity 1 .

\subsection{Group demand}

Following the standard collective approach (see Chiappori, 1988, 1992), we assume that group decisions are Pareto efficient. The demand function solves a program of the form:

$$
\max _{x_{1}^{1}, \ldots, x_{S}^{n}, X} A_{1}\left(x_{1}^{2}, \ldots, x_{1}^{n}, X\right)+x_{1}^{1} b(X)
$$

under the constraints

$$
\begin{gathered}
A_{m}\left(x_{m}^{2}, \ldots, x_{m}^{n}, X\right)+x_{m}^{1} b(X) \geq \bar{u}_{m}, m=2, \ldots, S, \\
\text { and } \\
\sum_{i, m} p^{i} x_{m}^{i}+\sum_{j} P^{j} X^{j}=\sum_{s} y_{s}=y
\end{gathered}
$$

Let $\mu_{m}$ denote the Lagrange multiplier of constraint $(4 \mathrm{~m})$, i.e. the Pareto weight of member $m$, and $\lambda$ the Lagrange multiplier of constraint (4). If $x_{m}^{1}>0$ for all $m$, first order conditions give $\lambda=1$ and $\mu_{m}=\lambda$ for all $m$; therefore $\mu_{m}=1$ and the program becomes:

$$
\max _{x_{1}^{1}, \ldots, x_{S}^{n}, X} \sum_{m} A_{m}\left(x_{m}^{2}, \ldots, x_{m}^{n}, X\right)+\left(\sum_{m} x_{m}^{1}\right) b(X)
$$

under the budget constraint. Defining the function $A$ by

$$
A\left(x^{2}, \ldots, x^{n}, X\right)=\max _{\left(x_{1}^{2}, \ldots, x_{1}^{n}, \ldots, x_{S}^{2}, \ldots, x_{S}^{n}\right)}\left\{\begin{array}{c}
\sum_{m} A_{m}\left(x_{m}^{2}, \ldots, x_{m}^{n}, X\right) \\
\text { s.t. } \sum_{m} x_{m}^{i}=x^{i}, i=2, \ldots, n
\end{array}\right\}
$$

we see that group demand can be derived from the maximization, subject to the family budget constraint, of a single utility function $U^{H}$ which is also of the generalized quasi-linear form:

$$
U^{H}\left(x^{1}, x^{2}, \ldots, x^{n}, X\right)=A\left(x^{2}, \ldots, x^{n}, X\right)+x^{1} b(X) .
$$

Equivalently, for any Pareto efficient allocation the vector $\left(x_{m}^{2}, \ldots, x_{m}^{n}, X\right)$ solves:

$$
\max _{x_{1}^{1}, \ldots, x_{S}^{n}, X} \sum_{m} A_{m}\left(x_{m}^{2}, \ldots, x_{m}^{n}, X\right)+\left(y-\sum_{i, m} p^{i} x_{m}^{i}-\sum_{j, m} P^{j} X^{j}\right) b(X),
$$


which shows that the choice of $\left(x_{m}^{2}, \ldots, x_{m}^{n}, X\right)$ is the same for all Pareto efficient outcomes; a particular Pareto efficient allocation is defined by the choice of the private good consumptions $x_{m}^{1}$.

Conversely, if a group demand stems from the maximization of a unique utility of the form (6), then it can always be derived as the Pareto efficient aggregate demand of a group satisfying the $T U$ property. Indeed, one can then define

$$
a\left(x_{m}^{2}, \ldots, x_{m}^{n}, X\right)=\frac{1}{S} A\left(S x_{m}^{2}, \ldots, S x_{m}^{n}, X\right)
$$

and

$$
u_{m}\left(x_{m}, X\right)=a\left(x_{m}^{2}, \ldots, x_{m}^{n}, X\right)+x_{m}^{1} b(X), m=1, \ldots, S .
$$

Note that $a$ is concave like $A$.

The $u_{m}$ define a group that satisfies the $T U$ property. Any Pareto efficient allocation for this group solves

$$
\max _{\left(x_{1}^{1}, \ldots, x_{1}^{n}, \ldots, x_{S}^{1}, \ldots, x_{S}^{n}, X\right)}\left(\sum_{m} a\left(x_{m}^{2}, \ldots, x_{m}^{n}, X\right)\right)+\left(\sum_{m} x_{m}^{1}\right) b(X)
$$

Now, consider the program:

$$
\max _{\left(x_{1}^{2}, \ldots, x_{1}^{n}, \ldots, x_{S}^{2}, \ldots, x_{S}^{n}\right)}\left\{\begin{array}{c}
\sum_{m} a\left(x_{m}^{2}, \ldots, x_{m}^{n}, X\right) \\
\text { s.t. } \sum_{m} x_{m}^{i}=x^{i}, i=2, \ldots, n
\end{array}\right\}
$$

By concavity of $a$, the solution is $x_{m}^{i}=x^{i} / S$ for all $i, m$, and the value of the program is thus

$$
\begin{aligned}
\sum_{m} a\left(\frac{x^{2}}{S}, \ldots, \frac{x^{n}}{S}, X\right) & =S \frac{1}{S} A\left(S \frac{x^{2}}{S}, \ldots, S \frac{x^{n}}{S}, X\right) \\
& =A\left(x^{2}, \ldots, x^{n}, X\right)
\end{aligned}
$$

therefore any Pareto efficient allocation must solve

$$
\max _{\left(x^{1}, \ldots, x^{n}, X\right)} A\left(x^{2}, \ldots, x^{n}, X\right)+x^{1} b(X)
$$

hence the conclusion.

Throughout the paper, we assume that $U^{H}$ is $C^{2}$ and strongly concave; equivalently, demand functions are assumed continuously differentiable.

\section{Testable implications of the GQL form}

We now study the properties of such demand functions. Namely, we address the following question. Assume that the behavior of a particular group can be described 
as the efficient demand generated by a TU context; equivalently, the group's demand is stemming from the maximization of a unique utility of the generalized quasi linear form (6). What testable restrictions does the form under consideration imply for observable behavior - i.e. demand functions? And are these restrictions (if any) 'likely' to be empirically fulfilled - in other words, how easy is it to empirically falsify the TU property? We now provide a comprehensive answer to these questions.

\subsection{Unitary conditions}

A first and obvious requirement of the $T U$ assumption is that household demand be derived from the maximization of a single utility, namely $U^{H}$. Therefore, the resulting household demand must satisfy the standard, 'unitary' restrictions reflecting this fact. Specifically, household demand must satisfy:

- Income pooling: it only depends on total income $y=\sum_{m} y_{m}$

- Slutsky symmetry and negativeness.

These conditions, by themselves, constrain the form of household demands; income pooling, in particular, has been rejected by numerous empirical studies. In practice, both individual and household demands must satisfy income pooling except for commodity 1; different Pareto efficient outcomes correspond to different allocations of this good. If individual incomes have an impact on the allocation, this will be reflected in the structure of individual demands for good 1.

In addition, the $T U$ assumption requires household utility to belong to a specific family, namely the generalized quasi linear one. We now investigate the additional restrictions generated by this requirement.

\subsection{Necessary conditions 1: conditional quasilinearity}

A first implication of form (6) is that the $U^{H}$, considered as a function of private consumptions only, is quasi linear. The implications of this facts are derived in the following two statements:

Proposition 3 Assume that $(x, X)$, as functions of $(p, P, y)$, stems from the maximization of some generalized quasi-linear utility $U^{H}$ under the budget constraint. Then in a neighborhood of a point $(p, P, y)$ such that $x^{1}(p, P, y)>0$, each $x^{i}, i=$ $2, \ldots, n$, can be written as a function of $(p, X)$ alone. In particular, the vector $D_{P, y} x^{i}=$ $\left(\frac{\partial x^{i}}{\partial P^{1}}, \ldots, \frac{\partial x^{i}}{\partial P^{N}}, \frac{\partial x^{i}}{\partial y}\right)^{\prime}$ can be written as a linear combination of $D_{P, y} X^{j}, j=1, \ldots, N$.

Proof. See Appendix. 
Proposition 3 generalizes the standard property of quasi-linear functions. If $X=$ 0 , indeed, it implies that $\partial x^{i} / \partial y=0$ for all $i \geq 2$, a property characteristic of quasilinearity. Clearly, the necessary condition in Proposition 3 is much less restrictive than quasi-linearity; it simply requires the existence of a specific link between the income effects of private and public goods.

Proposition 3 has a very natural interpretation. Namely, it expresses the fact that conditional on public goods, the group utility (as a function of private consumptions) is quasilinear. ${ }^{6}$ In particular, one can show the following: if the conditions in Proposition 3 are satisfied, then there exists two functions $\tilde{U}^{H}$ and $u^{H}$ such that:

$$
U^{H}\left(x^{1}, x^{2}, \ldots, x^{n}, X\right)=\tilde{U}^{H}\left[x^{1}+u^{H}\left(x^{2}, \ldots, x^{n}, X\right), X\right]
$$

Moreover, the function $u^{H}$ is uniquely identified from the group demand. The proof of this claim is in Appendix.

Aggregate consumption index Since the function $u^{H}$ is uniquely identified, we may define an 'aggregate consumption index' $x^{A}=x^{1}+u^{H}\left(x^{2}, \ldots, x^{n}, X\right)$. The condition in Proposition 3 implies that $U^{H}$ is a function of $\left(x^{A}, X\right)$ only, $U^{H}=\tilde{U}^{H}\left[x^{A}, X\right]$. In other words, private consumptions matter only through the index $x^{A}$. Note, however, that the index $x^{A}$ depends on both private and public consumptions. Finally, if $d$ denotes total household expenditures on private goods:

$$
d\left(p, X, x^{A}\right)=\min \left[\begin{array}{c}
\sum p^{i} x^{i} \\
\text { s.t. } x^{1}+u^{H}\left(x^{2}, \ldots, x^{n}, X\right)=x^{A}
\end{array}\right]
$$

the household program can be written as:

$$
\max \left[\begin{array}{c}
\tilde{U}^{H}\left[x^{A}, X\right] \\
\text { s.t. } d\left(p, X, x^{A}\right)+\sum P^{j} X^{j}=y
\end{array}\right]
$$

\subsection{Necessary conditions 2: additional restrictions}

The generalized quasi linear form imposes further restrictions on the form of the function $\tilde{U}^{H}$; namely, $\tilde{U}^{H}$ must be (up to an increasing transform) affine in the aggregate index $x^{A}$. This requirement has a direct implication for demand functions.

The corresponding conditions turn out to be easier to express in terms of the inverse demand function. Take some specific demand $(x, X)$, expressed as a function of $\left(p^{2}, \ldots, p^{n}, P^{1}, \ldots, P^{N}, y\right)$, and consider some open set $\mathcal{O}^{\prime}$ on which its Jacobian

${ }^{6} \mathrm{~A}$ function $u(x)$ is quasilinear if there exists two increasing functions $F$ and $\bar{u}$ such that:

$$
u(x)=F\left[x^{i}+\bar{u}\left(x^{-i}\right)\right] \text { for some } i
$$

In practice, one may, without loss of generality, assume that $i=1$. We adopt this convention throughout the paper. 
determinant does not vanish. Then we can invert it, thus defining the inverse demand function

$$
\begin{aligned}
p^{i} & =\pi^{i}(x, X), \quad i=2, \ldots, n \\
P^{k} & =\Pi^{k}(x, X), \quad k=1, \ldots, N \\
y & =\theta(x, X)
\end{aligned}
$$

This inverse demand functions must satisfy the following restrictions:

Proposition 4 Assume that $(x, X)$, as functions of $(p, P, y)$, stems from the maximization of some generalized quasi-linear utility $U^{H}$ under the budget constraint. Consider some open set $\mathcal{O}^{\prime}$ on which its Jacobian determinant does not vanish. The inverse demand function $(\pi, \Pi, \theta)$ satisfies the following conditions:

1. For any $1 \leq k \leq N, \Pi^{k}(x, X)$ has the affine (in $\left.x^{1}\right)$ form:

$$
\Pi^{k}(x, X)=\alpha_{k}\left(x^{-1}, X\right)+x^{1} \beta_{k}(X)
$$

for some functions $\alpha_{k}$ and $\beta_{k}$ (where $\left.x^{-1}=\left(x^{2}, \ldots, x^{n}\right)\right)$. In particular,

$$
\frac{\partial^{2} \Pi_{k}}{\partial x^{1} \partial x^{i}}=0, \quad i=1, \ldots, n
$$

2. For any $1 \leq k \leq N, 1 \leq j \leq N$,

$$
\frac{\partial \beta_{k}}{\partial X^{j}}=\frac{\partial \beta_{j}}{\partial X^{k}}
$$

3. If $b(X)$ is such that $\beta_{k}(X)=\frac{\partial \log b(X)}{\partial X^{k}}, k=1, \ldots, N$, the matrix $M$ of general term

$$
M_{k, j}=\frac{\partial\left[\alpha_{k}\left(x^{-1}, X\right) b(X)\right]}{\partial X^{j}}
$$

is symmetric:

$$
\frac{\partial\left[\alpha_{k}\left(x^{-1}, X\right) b(X)\right]}{\partial X^{j}}=\frac{\partial\left[\alpha_{j}\left(x^{-1}, X\right) b(X)\right]}{\partial X^{k}}
$$

Proof. See Appendix. Note that condition (11) guarantees the existence and the uniqueness (up to a multiplicative constant) of the function $b(X)$ used in condition (12). Indeed, from (11) the vector $\left(\beta_{1}, \ldots, \beta_{N}\right)^{\prime}$ is the gradient of some function $B(X)$, and we may then define $b(X)=\exp (B(X))$. 


\subsection{Sufficient conditions}

The conditions just derived are in fact sufficient for the GQL form. Specifically, we have the following result:

Proposition 5 Let $(x, X)$ be a demand function of $(p, P, y)$, satisfying Slutsky, and consider an open set $\mathcal{O}$ on which $x^{1}(p, P, y)>0$. Assume that in $\mathcal{O}$ :

- The vector $D_{P, y} x^{i}=\left(\frac{\partial x^{i}}{\partial P^{1}}, \ldots, \frac{\partial x^{i}}{\partial P^{N}}, \frac{\partial x^{i}}{\partial y}\right)^{\prime}$ can be written as a linear combination of $D_{P, y} X^{j}, j=1, \ldots, N$.

- Conditions (10), (11) and (12) of Proposition 4 are satisfied.

Then one can construct in $\mathcal{O}$ three functions $A, b$ and $\tilde{U}^{H}$ such that the demand is derived from a group utility of the form:

$$
U^{H}(x, X)=\tilde{U}^{H}\left[A\left(x^{-1}, X\right)+x^{1} b(X)\right]
$$

Proof. See Appendix.

\subsection{A linear expenditures example}

To illustrate the previous results, let us consider a simple example in a linear expenditure system (LES). A nice property of the LES system is that the underlying utility is well known, which allows to directly check the validity of the conclusions.

One private good We start with the case of one private good only $(n=1)$. Demands take the linear form:

$$
\begin{gathered}
x=c_{0}+c_{y} y+\sum_{j} c_{j} P^{j} \\
P^{k} X^{k}=\left(C_{0}^{k}+C_{y}^{k} y+\sum_{j} C_{j}^{k} P^{j}\right)
\end{gathered}
$$

with

$$
c_{y}+\sum C_{y}^{k}=1, c_{0}+\sum C_{0}^{k}=0, c_{j}+\sum_{k} C_{j}^{k}=0 \text { for all } j
$$

where the price of the private good $x$ has been normalized to 1 .

As it is well known, Slustky conditions require the coefficients to be proportional:

$$
\frac{C_{0}^{k}}{c_{o}}=\frac{C_{y}^{k}}{c_{y}}=\frac{C_{j}^{k}}{c_{j}} \text { for all } j \neq k
$$


so that the form is:

$$
\begin{gathered}
x=\gamma_{0}+c_{y}\left(y-\gamma_{0}-\sum_{j} \Gamma_{j} P^{j}\right) \\
P^{k} X^{k}=\Gamma_{k} P^{k}+C_{y}^{k}\left(y-\gamma_{0}-\sum_{j} \Gamma_{j} P^{j}\right)
\end{gathered}
$$

where

$$
\gamma_{0}=\frac{c_{0}}{1-c_{y}}, \Gamma_{j}=-\frac{c_{j}}{c_{y}}
$$

Since there is only one private good, the conditions of Proposition 3 are always satisfied. We now consider conditions (10), (11) and (12) of Proposition 4. The inverse demand function takes the form:

$$
\begin{aligned}
P^{k} & =\frac{C_{y}^{k}}{c_{y}} \frac{x-\gamma_{0}}{\left(X^{k}-\Gamma_{k}\right)} \\
y & =\frac{x-\gamma_{0}}{c_{y}}+\gamma_{0}+\sum_{j} \Gamma_{k} \frac{C_{y}^{k}}{c_{y}} \frac{x-\gamma_{0}}{\left(X^{k}-\Gamma_{k}\right)}
\end{aligned}
$$

so that (10) is satisfied with

$$
\alpha_{k}(X)=-\frac{C_{y}^{k} \gamma_{0}}{c_{y}\left(X^{k}-\Gamma_{k}\right)}, \beta_{k}(X)=\frac{C_{y}^{k}}{c_{y}\left(X^{k}-\Gamma_{k}\right)}
$$

In addition, $\frac{\partial \beta_{k}}{\partial X^{j}}=0$ for $k \neq j$, so that (11) are fulfilled as well, and

$$
b(X)=\prod\left(X^{k}-\Gamma_{k}\right)^{\frac{C_{y}^{k}}{c_{y}}}
$$

We now investigate (12). Here,

$$
\alpha_{k}(X) b(X)=-\frac{C_{y}^{k} \gamma_{0}}{c_{y}\left(X^{k}-\Gamma_{k}\right)} \prod\left(X^{s}-\Gamma_{s}\right)^{\frac{C_{y}^{s}}{c_{y}}}
$$

so that for $j \neq k$ :

$$
M_{k, j}=-\frac{C_{y}^{k} C_{y}^{j} \gamma_{0}}{c_{y}^{2}\left(X^{k}-\Gamma_{k}\right)\left(X^{j}-\Gamma_{j}\right)} \prod\left(X^{s}-\Gamma_{s}\right)^{\frac{C_{y}^{s}}{c_{y}}}
$$

which is symmetric. We conclude that the demand under consideration can always be derived from a GQL utility. One can easily show that the corresponding GQL utility is of the form:

$$
U(x, X)=\tilde{U}^{H}\left[x^{1} \prod\left(X^{k}-\Gamma_{k}\right)^{\frac{c_{y}^{k}}{c_{y}}}-\gamma_{0} \prod\left(X^{k}-\Gamma_{k}\right)^{\frac{c_{y}^{k}}{c_{y}}}\right] .
$$


General case We now study the general case of $n$ private goods, $n \geq 1$. The LES framework gives:

$$
\begin{gathered}
x=\gamma_{0}+c_{y}\left(y-\gamma_{0}-\sum \gamma_{i} p^{i}-\sum_{j} \Gamma_{j} P^{j}\right) \\
p^{i} x^{i}=\gamma_{i} p^{i}+c_{y}^{i}\left(y-\gamma_{0}-\sum \gamma_{i} p^{i}-\sum_{j} \Gamma_{j} P^{j}\right) \\
P^{k} X^{k}=\Gamma_{k} P^{k}+C_{y}^{k}\left(y-\gamma_{0}-\sum \gamma_{i} p^{i}-\sum_{j} \Gamma_{j} P^{j}\right)
\end{gathered}
$$

and we need to assume that $c_{y}^{i} \neq 0$ for all $i$ and $C_{y}^{j} \neq 0$ for all $j$.

In particular:

$$
D_{P, y} x^{i}=\left(-\frac{c_{y}^{i} \Gamma_{1}}{p^{i}}, \ldots,-\frac{c_{y}^{i} \Gamma_{N}}{p^{i}}, \frac{c_{y}^{i}}{p^{i}}\right)^{\prime}
$$

and

$$
D_{P, y} X^{k}=\left(-\frac{C_{y}^{k} \Gamma_{1}}{P^{k}}, \ldots,-\frac{C_{y}^{k} \Gamma_{k}}{P^{k}}-\frac{C_{y}^{k}}{\left(P^{k}\right)^{2}}\left(y-\gamma_{0}-\sum \gamma_{i} p^{i}-\sum_{j} \Gamma_{j} P^{j}\right), \ldots,-\frac{C_{y}^{k} \Gamma_{1}}{P^{k}}, \frac{C_{y}^{k}}{P^{k}}\right)
$$

It follows that the determinant

$$
D=\left|D_{P, y} x^{i}, D_{P, y} X^{1}, \ldots, D_{P, y} X^{N}\right|
$$

can be written as:

$$
D=\left|\begin{array}{ccc}
-\frac{c_{y}^{i} \Gamma_{1}}{p^{i}} & -\frac{C_{y}^{1} \Gamma_{1}}{P^{1}}-\frac{C_{y}^{1}\left(y-\gamma_{0}-\sum \gamma_{i} p^{i}-\sum_{j} \Gamma_{j} P^{j}\right)}{\left(P^{1}\right)^{2}} & -\frac{C_{y}^{N} \Gamma_{1}}{P^{N}} \\
-\frac{c_{y}^{i} \Gamma_{2}}{p^{i}} & -\frac{C_{y}^{1} \Gamma_{2}}{P^{1}} & -\frac{C_{y}^{N} \Gamma_{2}}{P^{N}} \\
-\frac{c_{y}^{i} \Gamma_{N}}{p^{i}} & -\frac{C_{y}^{1} \Gamma_{N}}{P^{1}} & -\frac{C_{y}^{N} \Gamma_{N}}{P^{N}}-\frac{C_{y}^{N}\left(y-\gamma_{0}-\sum \gamma_{i} p^{i}-\sum_{j} \Gamma_{j} P^{j}\right)}{\left(P^{N}\right)^{2}} \\
\frac{c_{y}^{i}}{p^{i}} & \frac{C_{y}^{1}}{P^{1}} & C^{N}
\end{array}\right|
$$

Factoring out the common factors and substracting the first column from each other, we find that $D$ is zero if and only if

$$
D^{\prime}=\left|\begin{array}{ccc}
\Gamma_{1} & -\frac{y-\gamma_{0}-\sum \gamma_{i} p^{i}-\sum_{j} \Gamma_{j} P^{j}}{P^{1}} & 0 \\
\Gamma_{2} & 0 & 0 \\
& & 0 \\
\Gamma_{N} & 0 & -\frac{y-\gamma_{0}-\sum \gamma_{i} p^{i}-\sum_{j} \Gamma_{j} P^{j}}{P^{N}} \\
1 & 0 & 0
\end{array}\right|=0
$$


which is impossible since

$$
D^{\prime}=\frac{\left(y-\gamma_{0}-\sum \gamma_{i} p^{i}-\sum_{j} \Gamma_{j} P^{j}\right)^{n}}{\prod_{k} P^{k}} .
$$

Therefore, the vector $D_{P, y} x^{i}$ cannot be a linear combination of the $\left(D_{P, y} X^{k}, k=1, \ldots, N\right)$. We conclude that the conditions of Proposition 3 cannot be satisfied; the LES form is not compatible with GQL unless there is exactly one private good.

\section{Conclusion}

A transferable utility framework is bound to generate strong predictions and lead to clear-cut policy recommendation regarding important economic and social issues. For instance, the Beckerian analysis of the marriage market suggests that when designing possible reforms of divorce laws, governments should not consider the impact on the number of divorces, since such an impact should be nil in any case. Various versions of the Coase theorem have been extensively used in Law and Economics; again, the TU assumption plays a crucial role in their validity. In almost all cases, though, utilities are assumed to be transferable without much justification, and no empirical test is performed to verify the empirical relevance of this assumption. Indeed, not much is known on how to test for TU.

The main goal of this note was to fill this gap, by deriving testable implications of the Transferable Utility assumption. Our first conclusion is that the empirical relevance of the TU assumption largely depend on the structure of intragroup consumption. When all commodities are privately consumed, then TU is known to be equivalent to quasilinear utilities, which requires zero income elasticity for all demands but one. On the contrary, should some consumption be public within the group, TU is observationally equivalent to the household maximizing a generalized quasi linear utility (GQL) a la Bergstrom (1989). ${ }^{7}$

We derive a set of necessary and sufficient conditions on demand that fully characterize GQL utilities. These conditions are of two sorts. First, the 'unitary' requirement that household behaves a single utility maximizer generates standard restrictions (income pooling, Slutsky). Additional restriction come from the GQL form; they require price variations to be available in the data set used for empirical testing. More surprising is the fact that the conditions only depend on the prices of the public goods; i.e., the GQL form has no (direct) bite on the structure of private price elasticities. A consequence of this remark is that the GQL form cannot be tested without variations in the prices of public commodities. If the only data available are

\footnotetext{
${ }^{7}$ Some versions of the Coase theorem require the TU property to hold in different institutional contexts. For instance, Becker's analysis of divorce assumes TU both before and after divorce. This requirement may be hard to reconcile with the public nature of some consumptions; see Chiappori, Iyigun and Weiss (2006) for a detailed analysis.
} 
cross sectional, or if the prices of the public goods are not available (as is often the case with durables), the GQL form is simply not testable from empirical demands. In the opposite case, the restrictions can be tested using standard economic tools, a task that is left for future research. 


\section{APPENDIX}

\section{A Proof of Proposition 3}

Assume that $U^{H}$ is generalized quasi linear. When $x^{1}>0$, first-order conditions give

$$
\frac{\partial A}{\partial x^{i}}=\lambda p^{i}, i=2, \ldots, n \text { and } \quad b(X)=\lambda .
$$

where $\lambda$ is the Lagrange multiplier of the budget constraint. Therefore

$$
\frac{\partial A\left(x^{2}, \ldots, x^{n}, X\right)}{\partial x^{i}}=p^{i} b(X), i=2, \ldots, n .
$$

These $(n-1)$ equations pin down the $(n-1)$ unknowns $x^{2}, \ldots, x^{n}$. It follows that each $x^{i}$ can be written as a function of $p$ and $X$ alone. The second part follows immediately.

\section{B Proof of Proposition 4}

Consider the program

$$
\max \left\{\begin{array}{c}
A\left(x^{2}, \ldots, x^{n}, X\right)+x^{1} b(X) \\
\text { under } x^{1}+\sum_{i \geq 2} p^{i} x^{i}+\sum_{k} P^{k} X^{k}=y
\end{array}\right\}
$$

Assuming, as above, $x^{1}>0$, we get the following first order conditions:

$$
\frac{\partial A}{\partial X^{k}}+x^{1} \frac{\partial b}{\partial X^{k}}=\lambda P^{k}, i=2, \ldots, n \text { and } \quad b(X)=\lambda .
$$

where $\lambda$ is the Lagrange multiplier of the budget constraint. Therefore

$$
\frac{\partial A / \partial X^{k}}{b(X)}+x^{1} \frac{\partial b / \partial X^{k}}{b(X)}=P^{k}
$$

which gives (10) for

$$
\alpha_{k}\left(x^{-1}, X\right)=\frac{\partial A\left(x^{-1}, X\right) / \partial X^{k}}{b(X)} \text { and } \beta_{k}(X)=\frac{\partial b(X) / \partial X^{k}}{b(X)}
$$

This form implies, furthermore, that the vector $\left(\beta_{1}, \ldots, \beta_{N}\right)^{\prime}$ be a gradient:

$$
\beta_{k}(X)=\frac{\partial \log b(X)}{\partial X^{k}}
$$

and (11) follows. Finally, on $\{\beta\}^{\perp}, b(X)$ is constant, so $\alpha_{k}\left(x^{-1}, X\right)$ is a gradient, implying (12). 


\section{Proof of Proposition 5}

In what follow, we normalize $p^{1}$ to be 1 . The proof in is three steps.

Step 1: conditional quasilinearity We first show the following result: if $(x, X)$ be a demand function of $(p, P, y)$, satisfying Slutsky, and such that, on some open set $\mathcal{O}$

- $x^{1}(p, P, y)>0$

- the vector $D_{P, y} x^{i}=\left(\frac{\partial x^{i}}{\partial P^{1}}, \ldots, \frac{\partial x^{i}}{\partial P^{N}}, \frac{\partial x^{i}}{\partial y}\right)^{\prime}$ can be written as a linear combination of $D_{P, y} X^{j}, j=1, \ldots, N$,

then one can construct in $\mathcal{O}$ two functions $u^{H}$ and $\tilde{U}^{H}$ such that the demand is derived from a group utility of the form:

$$
U^{H}\left(x^{1}, x^{2}, \ldots, x^{n}, X\right)=\tilde{U}^{H}\left[x^{1}+u^{H}\left(x^{2}, \ldots, x^{n}, X\right), X\right]
$$

Moreover, the function $u^{H}$ is uniquely identified from the group demand.

The proof relies on the following Lemma:

Lemma 6 A direct utility $u(x)$ is quasilinear if and only if the corresponding expenditure function e satisfies, for u large enough:

$$
\frac{\partial e(p, u)}{\partial u}=p^{1} \phi\left(p^{1} u\right)
$$

for some increasing function $\phi$. In particular, if we choose to normalize $p^{1}$ to 1 , we have

$$
\frac{\partial e(p, u)}{\partial u}=\phi(u)
$$

and $\frac{\partial e(p, u)}{\partial u}$ does not depend on prices.

Proof. of the Lemma: If $u(x)$ is quasilinear, the corresponding indirect utility $v$ is of the form:

$$
v(p, y)=F[y-\bar{v}(p)]
$$

Inverting in $y$ gives the expenditure function:

$$
e(p, u)=\Phi(u)+\bar{v}(p)
$$

where $\Phi=F^{-1}$. The conclusion follows. Conversely, if (21) holds, then (24) and (23) follow. Consider the program

$$
w(x)=\min _{p, y}\left\{y-\bar{v}(p) \text { s.t. } \sum_{i} p^{i} x^{i}=y\right\}
$$

From the envelope theorem, $\frac{\partial w}{\partial x^{1}}=\lambda$, where $\lambda$ is the Lagrange multiplier of the budget constraint. Form the first order condition in $y, \lambda=1$. It follows that $\frac{\partial w}{\partial x^{1}}=1$, therefore $w(x)=x^{1}+\bar{u}\left(x^{-1}\right)$ for some $\bar{u}$, and finally $u=F(w)$. 
We may now proceed. Consider, now, some demand $(x, X)$ such that $x^{1} \neq 0$ on some open set, and assume that each $x^{i}, i=2, \ldots, n$, can be written as a function of $(p, X)$ alone. For some given $\bar{X}$, consider the function

$$
u^{H}\left(x^{1}, x^{2}, \ldots, x^{n}\right)=U^{H}\left(x^{1}, x^{2}, \ldots, x^{n}, \bar{X}\right)
$$

and let $v^{H}$ and $e^{H}$ be the corresponding indirect utility and expenditures functions:

$$
\begin{aligned}
& v^{H}\left(p^{2}, \ldots, p^{n}, \rho\right)=\max _{x}\left\{u^{H}\left(x^{1}, x^{2}, \ldots, x^{n}\right) \text { s.t. } x^{1}+\sum_{i \geq 2} p^{i} x^{i}=\rho\right\} \\
& e^{H}\left(p^{2}, \ldots, p^{n}, u\right)=\min _{x}\left\{x^{1}+\sum_{i \geq 2} p^{i} x^{i} \text { s.t. } u^{H}\left(x^{1}, x^{2}, \ldots, x^{n}\right)=u\right\}
\end{aligned}
$$

If $x\left(p^{2}, \ldots, p^{n}, \rho\right)$ is the Marshallian demand associated with $u^{H}$ (so that $x\left(p^{2}, \ldots, p^{n}, \rho\right)$ solves the right hand side of (25)), we have, by assumption:

$$
\frac{\partial x^{i}}{\partial \rho}=0 \text { for } i \geq 2, \text { and } \frac{\partial x^{1}}{\partial \rho}=1
$$

Therefore, if $\xi(p, u)$ denotes the Hicksian demand associated with $u^{H}$ (so that $\xi\left(p^{2}, \ldots, p^{n}, u\right.$ ) solves the right hand side of $(26))$, the identity $\xi^{i}\left(p^{2}, \ldots, p^{n}, u\right)=x^{i}\left[p^{2}, \ldots, p^{n}, e^{H}\left(p^{2}, \ldots, p^{n}, u\right)\right]$ gives:

$$
\frac{\partial \xi^{i}}{\partial u}=0 \text { for } i \geq 2, \text { and } \frac{\partial \xi^{1}}{\partial u}=\frac{\partial e^{H}}{\partial u}
$$

From

$$
\xi^{i}(p, u)=\frac{\partial e^{H}}{\partial p^{i}}
$$

we get

$$
\frac{\partial \xi^{i}(p, u)}{\partial u}=\frac{\partial^{2} e^{H}}{\partial p^{i} \partial u}=0 \text { for } i \geq 2
$$

which implies that $\frac{\partial e^{H}}{\partial u}$ is independent of prices. From the Lemma, we conclude that $u^{H}$ is quasi linear.

Finally, demand identifies $u^{H}$ up to an increasing transform, but only one (at most) of such transforms is such that $\partial u^{H} / \partial x^{1}=1$.

Step 2: additional conditions We now consider the conditions(10), (11) and (12) of Proposition 4. Assume they are satisfied. From (11), there exists some $B(X)$, defined up to a additive constant, the gradient of which is the vector $\left(\beta_{1}, \ldots, \beta_{N}\right)^{\prime}$. We may then define $b(X)=\exp (B(X))$, and $b$ is defined up to a multiplicative constant. (12) then implies the existence of some $A$ such that $\alpha_{k}\left(x^{-1}, X\right)=\frac{\partial A\left(x^{-1}, X\right) / \partial X^{k}}{b(X)}$ for all $k$. 
Since the demand satisfies Slutsky, it can be derived from the maximization of some utility $U^{H}$, and we know that:

$$
\frac{\partial U^{H} / \partial X^{k}}{\partial U^{H} / \partial x^{1}}=P^{k}
$$

Therefore $U^{H}$ must satisfy the set of partial differential equations:

$$
\frac{\partial U^{H} / \partial X^{k}}{\partial U^{H} / \partial x^{1}}=\frac{\partial A / \partial X^{k}}{b(X)}+x^{1} \frac{\partial b / \partial X^{k}}{b(X)}, k=1, \ldots, N
$$

Integrating, we get that:

$$
U^{H}(x, X)=F\left[A\left(x^{-1}, X\right)+x^{1} b(X), x^{-1}\right]
$$

for some increasing mapping $F$.

Finally, since from (20):

$$
U^{H}(x, X)=\tilde{U}^{H}\left[x^{1}+u^{H}\left(x^{-1}, X\right), X\right]
$$

we conclude that

$$
U^{H}(x, X)=\tilde{U}^{H}\left[A\left(x^{-1}, X\right)+x^{1} b(X)\right]
$$

which closes the proof. 


\section{References}

[1] Becker, G. S. (1992). "Fertility and the Economy," Journal of Population Economics, 5, 158-201.

[2] Becker G. (1993). Treatise on the Family, paperback edition, Harvard University Press.

[3] Browning, M., P. A. Chiappori, and Y. Weiss. (in progress). Family Economics, Tel Aviv University, unpublished textbook manuscript.

[4] Bergstrom, T. and R. Cornes. (1983). "Independence of Allocative Efficiency from Distribution in the Theory of Public Goods," Econometrica, 51, 1753-1765.

[5] Bergstrom, T. (1989). "A Fresh Look at the Rotten Kid Theorem-and Other Household Mysteries", Journal of Political Economy, Vol. 97, No. 5, pp. 1138-1159

[6] Chiappori, P. A. (1988). "Rational Household Labor Supply," Econometrica, 56, 63-90.

[7] Chiappori, P. A. (1992). "Collective Labor Supply and Welfare," Journal of Political Economy, 100 (3), 437-67.

[8] Chiappori, P. A. and I. Ekeland (2006), "The micro economics of group behavior: General characterization", Journal of Economic Theory, 130 1, 1-26.

[9] Chiappori, P. A. and I. Ekeland (2007), "The micro economics of group behavior: Identification", working paper, Columbia University.

[10] Chiappori, P. A., M. Iyigun, and Y. Weiss. (2006). "The Becker-Coase Theorem, Transferable Utility and Marital Instability," Tel Aviv University, unpublished manuscript.

[11] Coase, R. H. (1960). "The Problem of Social Cost," Journal of Law and Economics, 3, 1-23.

[12] A. Roth and M. Sotomayor, Two-Sided Matching, Econometric Society Monographs, Cambridge University Press, 2003 